\title{
Computer-Aided Verification of Software Process Model Properties *
}

\author{
Alfred Bröckers ${ }^{1}$ and Volker Gruhn ${ }^{2}$ \\ 1 AG Software Engineering, University of Kaiserslautern \\ Kaiserslautern, Germany \\ broecker@informatik.uni-kl.de \\ ${ }^{2}$ Fraunhofer-Institut für Software und Systemtechnik \\ c/o University of Dortmund \\ Dortmund, Germany \\ gruhn@udo.informatik.uni-dortmund.de
}

\begin{abstract}
In this paper we introduce an approach to computer-aided verification of software process model properties. This approach uses a net-based software process modeling language called FUNSOFT nets. FUNSOFT nets are highlevel Petri nets which are adapted to the application domain of software process modeling. In this paper we focus on the verification of static and dynamic software process model properties. We define some software process model properties and introduce the notion of coverability trees for FUNSOFT nets. Moreover, we discuss how a verification component for FUNSOFT nets is integrated into the software process management environment MELMAC.
\end{abstract}

\section{Keywords}

software process model verification, FUNSOFT nets, static and dynamic software process model properties, coverability trees, MELMAC

\section{Introduction}

Software process modeling is an area that has attracted increasing interest over the past few years. Some of the first papers explicitly addressing software processes as a subject of interest are [Leh87, Ost87]. Since then several workshops [Kat90, Per91, Fug91, Der92] and a conference [Dow91] have been devoted to software processes.

Many misunderstandings related to software process modeling in the late $80 \mathrm{~s}$ were due to terminology (software process modeling versus process programming, software process models versus life cycle models, project management versus software process management). Some of the key terms (such as software process, software process model, software process modeling language) are agreed in the software process community in the meantime. These terms are introduced below:

\footnotetext{
* The work described here was done while both authors were supported by the chair for software technology at the University of Dortmund.
} 
A software process consists of the set of activities performed during software development, including their scheduling, and the objects manipulated. This idea of a software process means that there is one software process for each software system developed. Several software processes can be driven by the same software process model. A software process model describes general features of a class of processes, but not those features which are specific to individual processes. Software process models describe which activities have to be performed by whom, who is allowed to access which documents, when particular activities have to be performed, and which tools are used within which activities. If a software process model is used to guide a software process, we say that the software process model is enacted [Dow86]. A language suitable for describing software process models is called a software process modeling language.

In contrast to software process models, life cycle models are very coarse-grained. Generally speaking, life cycle models do not describe activities that can be carried out by individual persons or teams of persons, but they structure software processes in coarse-grained units, referred to as phases or steps. Some life cycle models are the waterfall model [Roy70], the spiral model [Boe88], and prototyping models [Luq89].

The aim to describe software processes explicitly (and thereby to use software process models) is not new. Each company and each project has an idea of its way to carry out a software process. Kellner points out that 'narrative descriptions have long been employed by organizations to record their standard operating procedures' [Kel88]. Guidelines and advice summed up in manuals belong to this kind of software process model. Other narrative descriptions of software process models are represented by standards, laws, and policies.

Natural language is not the only means used to describe software process models. Various kinds of graphic languages are also used to model software processes. Several of these languages are used to describe project plans, configuration plans, and interaction diagrams. Well-known examples are PERT diagrams [Som92] and Gantt charts [LH89]. The mentioned languages suffer from being informal. Moreover, they focus on only a few aspects of software processes, while they do not allow to model software processes with all their facets.

Dealing with software processes in a systematic way (also called software process management in the following) promises to improve the quality of software and to increase of software developer's productivity. These benefits are based on:

Software process modeling By modeling software processes, people can understand software processes, they are able to discuss them, and they can find out if they have a common understanding of their current software process.

Software process analysis Explicitly described software processes are amenable to software process analysis. Analysis helps to prove software process properties and to improve process models on the basis of analysis results.

Software process enaction Processes can be enacted on the basis of software process models, if the software process modeling language used is formally defined. If a process is guided by its model, then it is ensured that the process does not violate the prescriptions formulated in the model. Moreover, software process enaction provides assistance and help to software developers and it frees them from menial 
work which can be done automatically.

While software process modeling and software process enaction have been discussed in various papers and while entire workshops have been devoted to these subjects [Dow86, Kat90], software process analysis has not been discussed to such an extent.

Amongst the very few software process modeling approaches that deal with software process model analysis, we find some dealing with evaluating already finished software processes [BR87], some focusing on software process simulation [BFG91], and some dealing with consistency checking for software process models [Kel89]. Two of the most advanced analysis approaches are the DesignNets approach [LH89] and the STATEMATE approach [Kel89]. These approaches check rudimentary software process model consistency and completeness and investigate some static software process model properties.

In this paper we emphasize the importance of software process model analysis in general and the benefits of verifying software process properties in particular. Verification of process model properties and process model modifications based on verification results help to improve software process models. This contributes to reaching higher degrees of process maturity [Hum89].

The analysis approach for FUNSOFT nets is process-centered, i.e. we do not concentrate on the analysis of software process results (such as source code, design documents, and technical documentation), but on properties of the software process itself. This is different from document-centered analysis approaches [BR87], where software process results are the focus of analysis.

Our approach to software process model analysis is based on the software process modeling language FUNSOFT nets. FUNSOFT nets are high level Petri nets which are adapted to the application domain of software process modeling. The semantics of FUNSOFT nets is formally defined in terms of Predicate/Transition ( $\operatorname{Pr} / \mathrm{T}$ nets) nets [EG91].

The FUNSOFT net approach to software process model analysis supports:

Software process validation Software process validation means to check how software processes are expected to behave. Software process validation is based on simulating software processes [DG91, Gru92, Gru].

Software process verification Verification of software process properties means to prove whether all processes based on one process model will or will not have certain properties.

Software process post-evaluation In contrast to validation and verification techniques, post-evaluation focuses on improving a software process model after enacting it [GS92]. That means, the records of an already finished software process are evaluated in order to find out where improvement is needed.

The relationship between these three branches of software process analysis is illustrated in Figure 1. Figure 1 shows that validation and verification are a-priori techniques (while post-evaluation is an a-posteriori technique) and that validation and postevaluation are experimental techniques (while verification is a mathematical technique). In this paper we restrict ourselves to verification facilities for FUNSOFT nets. 


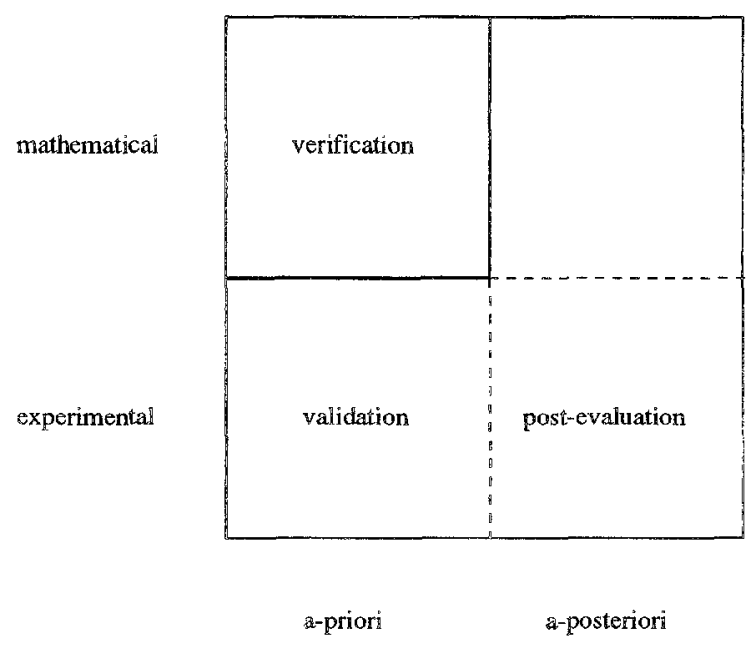

Fig. 1. Branches of software process model verification

The organization of this paper is as follows: In section 2 we introduce FUNSOFT nets. Section 3 introduces different kinds of verifiable software process model properties. In section 4 we discuss the architecture of the verification component for FUNSOFT nets and we explain how this component is integrated into the software process management environment MELMAC. Finally, section 5 concludes this paper with pointing out the focus of our future research on FUNSOFT net analysis and application of FUNSOFT net analysis facilities.

\section{An Introduction to FUNSOFT Nets}

The basic motivation for developing FUNSOFT nets was the finding that high level Petri nets, such as coloured Petri nets [Jen83, Jen87] and Pr/T nets [Gen87], provide analysis facilities that are worth exploiting in software process model analysis. However, none of these Petri net types fulfills all the key requirements for software process modeling languages such as integration of software development tools, modeling of complex object types, or representation of human interaction.

Another major drawback of standard high-level Petri net types is that they tend to yield rather large software process model representations. In contrast, FUNSOFT nets allow a much more dense representation of software process models. The number of nodes of a FUNSOFT net representing a software process model is an order of magnitude smaller than the number of nodes of the Pr/T net representing that model.

\subsection{An Informal Explanation of FUNSOFT Concepts}

Software process modeling based on FUNSOFT nets [GJ92] and software process enaction based on FUNSOFT [DG90] nets have been discussed elsewhere. In this paper 
we restrict ourselves to software process analysis. That is why we explain only those FUNSOFT net features absolutely necessary for understanding the proposed analysis facilities. For a complete discussion of FUNSOFT nets we refer to [Gru91b].

A FUNSOFT net contains a triple $(S, T ; F)$ which denotes a Petri net [Rei86]. Elements of $S$ are called channels. Channels are used to store objects, such as reports, test plans, specification documents. Elements of $T$ are called agencies. Agencies represent activities to be carried out in software processes such as design, edit, implement, review report. Elements of $F$ are called edges. Edges describe the relation between agencies and channels ${ }^{3}$.

Agencies can be refined. Agency refinement is the basis of structuring FUNSOFT nets hierarchically. By agency refinement it is possible to embed a high level activity like requirements analysis into a high level representation of a software process model, while details of the requirements analysis activity are hidden in the refinement. This notion of refinement corresponds to the notion of transition substitution [HJS89].

In the following we discuss the graphical representation of FUNSOFT nets. This discussion is related to the FUNSOFT net example presented in Figures 3-6. It is used to illustrate the graphical representation of FUNSOFT nets and the verification facilities for FUNSOFT nets. This example is in part taken from the Core Problem [Kat90] which is the reference example of the 6th International Software Process Workshop [Hei91].

Agencies are represented as rectangles. Each agency is annotated with a string containing the agency name followed by a colon and by the activity attached to that agency. In the second refinement level presented in Figure 5 we recognize, for example, an agency IdentComps to which the activity DecList is attached. Refined agencies are represented as shaded rectangles. Channels are represented as circles. Each channel is annotated with a string containing the channel name followed by a colon and by the object type attached to that channel. The top leftmost channel of the top level displayed in Figure 3 is called SystemReqs and associated with the object type LifeCycleDoc. Edges are represented as directed arcs.

Obviously, the information displayed in Figures 3-6 is only the skeleton of a software process model. It is not sufficient for defining a software process. Additionally, it is necessary to define types of objects stored in channels and activities attached to agencies. In the following we characterize these additional components of FUNSOFT nets and some attributes of channels, agencies, and edges. Moreover, we define the notion of FUNSOFT net markings, and, finally the fring rule for FUNSOFT nets.

Object types A FUNSOFT net contains a set of object types $O$. This set defines the object types that can be used in software process models. The types boolean, integer, real, string, text, and binary are predefined. Other object types can be constructed by using the construction operator struct (for building records) or list (for building lists). Object types are specified using a C-like syntax.

An example of an object type definition is given below.

SourceDoc $=$ struct $\{$ string name;

${ }^{3}$ Let $s$ be channel, let $t$ be an agency, $s$ is called a preset channel of $t$, if an edge $(s, t)$ exists. If an edge $(t, s)$ exists, $s$ is called a postset channel of $t$. The postset of an agency $t$ is denoted by $t \bullet$, its preset is denoted by $\bullet$. 


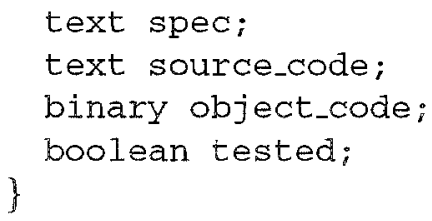

Activities A FUNSOFT net contains a set of activities $J$. Each activity $j$ has an input and an output parameterization, an input firing behavior, $F_{I N}(j) \in\{a l l, m u l t\}$, and an output firing behavior, $F_{\text {OUT }}(j) \in\{a l l$, det, mult, complex $\}$. The parameterizations describe the types of input and output parameters. The firing behaviors specify how many objects of each parameter type are consumed (input firing behavior), respectively produced (output firing behavior) in each execution of the activity.

We distinguish two input firing behaviors, namely all and mult:

- The input fring behavior all of an activity $j$ indicates that $j$ requires one input parameter per input parameter type. Thus, it implements the input firing behavior of transitions in standard Petri nets. The activity $C C$, for example (see below) has the input firing behavior all which means that it needs exactly one object of type SpecDoc in each firing.

- The input firing behavior mult of an activity $j$ indicates that $j$ needs a natural number $n$ as first input object, and $n$ input parameters of the second parameter type. Thus, the value of the first input parameter determines the number of consumed objects of the second parameter type. Exactly one input parameter is needed for any further input parameter type. An example of an activity with a mult input firing behavior is the activity of composing a list out of list elements. Such an activity requires a natural number as the first input parameter. This number determines how many list elements (corresponding to the second parameter type) are composed.

The output firing describes the different results the execution of the activity can have. We distinguish four output firing behaviors, namely all, det, mult, complex:

- The output firing behavior all of an activity $j$ means that $j$ produces one object per output parameter type in each execution. This corresponds to the input firing behavior of transitions in standard Petri nets.

- The output firing behavior det means that exactly one object is produced, but that it depends on values of input parameters an object of which type is produced.

- The output firing behavior mult of an activity $j$ indicates that $j$ produces a natural number $n$ as first output parameter, $n$ output parameters of the second output parameter type and one output parameter of any further output parameter type. An example of an activity with a mult output firing behavior is the DecList-activity. This activity decomposes a list into its elements (second output parameter type) and its length (first output parameter type). An activity with mult output firing behavior is associated with a range attribute. This attribute specifies how many objects of the second parameter type can be produced in one execution of the activity. 
- The output firing behavior complex of an activity $j$ means that there are several potential results of executing $j$, where each result could mean that several objects of different output parameter types are produced. An example of an activity with a complex output firing behavior is the $C C$-activity (representing a compilation). The result of a compilation is either a failure report and a specification document that has to be re-edited or a successfully compiled source code. Thus, the output firing behavior of the $C C$-activity is specified by the term (1 AND 2) XOR 3.

Moreover, an activity is described by its automation mode. An activity $j$ with automation mode $A U T O$ can be executed automatically, i.e. without human interaction. An individual interaction activity $j$ (automation mode $=M A N$ ) requires interaction of one software developer. A social interaction activity $j$ (automation mode $=M A N Y$ ) requires the interaction of several software developers with each other.

\section{ACTIVITY_NAME : CC \\ INFORMAL_DESCRIPTION : \\ The activity $C C$ reads an object of the type SourceDoc (whose object code part is empty) and produces either a text (corre- sponding to a failure report) and an object of type SourceDoc (with empty object code part) which is to be re-edited or an object of type SourceDoc (whose object code part is not empty). It is based on the tool $c c$ under SUN OS 4.0.}

PARAMETERIZATION:

IN: SourceDoc

OUT: text

SourceDoc

SourceDoc

FIRING_BEHAVIOR:

IN : all

OUT: complex (1 AND 2) XOR (3)

AUTOMATION MODE:

AUTO

The head of the $C C$-activity given above is complemented by a simulation description and by an execution description. These two parts of an activity are described in the $\mathrm{C}$ programming language. The simulation description is executed in simulating a $C C$-agency, the execution description is executed in firing a $C C$-agency in a software process. These two parts are omitted here. Examples of simulation and execution descriptions of activities can be found in [DG90].

Channel attributes $S_{T}$ attaches an object type from $O$ to each channel. Each channel $s$ can only be marked with objects of type $S_{T}(s)$.

Agency attributes The functions $T_{J}$ and $T_{T}$ assign attributes to agencies. 


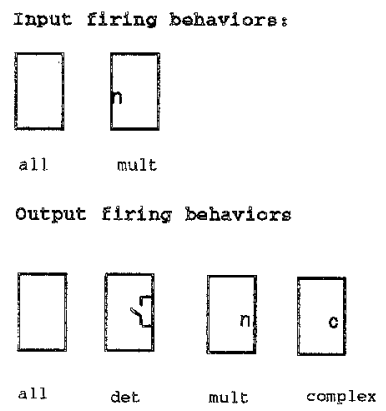

Fig. 2. Graphical representations of input and output firing behaviors

$T_{J}(t)$ denotes the activity assigned to agency $t$. When an agency is fired (see firing rule below), the associated activity is executed ${ }^{4}$. The input and output firing behaviors of an activity $j$ determine the graphical representation of an $j$-agency. Figure 2 displays the representations of the firing behaviors mentioned above. Input and output firing behaviors are independent from each other. Accordingly all combinations of input firing behavior symbols and output firing behavior symbols are possible.

The function $T_{T}$ assigns a non-negative real value to agencies. $T_{T}(t)$ denotes the time consumption of agency $t$. It quantifies the amount of time which passes between reading objects from the preset of $t$ and writing objects to the postset of $t$ during software process simulation.

Edge attributes The function $F_{T}$ assigns an edge type to each edge. The most important edge types are $O U$ (OUtput) for edges between agencies and channels, and $I N$ (INput) and $C O$ (COpy) for edges between channels and agencies. When an object is read via an $I N$-edge it is removed from the channel it was stored in. Reading an object via an $\mathrm{CO}$-edge means to copy it. Further edge types are used to describe control flow. An edge of type ST (STart) represents a control flow edge from channel to agency. An edge of type $F I$ (FInish) represents a control flow edge from agency to channel. A further function is the edge numbering function $F_{N}$, used to define an order of pre- and postset channels of each agency. This order is needed to distinguish channels being associated with the same parameter type in the preor postset of an agency. In the rest of this paper we assume that pre- and postset channels of agencies are ordered according to their index, i.e. $s_{1}$ is the first preset channel, $s_{2}$ the second etc.

Marking The initial marking of the net is defined by the function $M$, which assigns a set of objects of $S_{T}(s)$ to each channel $s$.

Firing rule The firing rule for FUNSOFT nets defines when agencies can be fired and how the number of objects in channels is manipulated when an agency actually is fired. The firing rule only relates to numbers of objects, but not to their values. The values of objects depend on the activity to be executed when an agency is fired.

\footnotetext{
${ }^{4}$ An agency associated with an activity $x y$ is called an $x y$-agency in the following.
} 
The object manipulation by activities is not subject of the firing rule. The firing rule explanation is subdivided into four parts. In the first part we explain when an agency can be fired (this depends on the input firing behavior of the activity associated with the agency, compare explanation of input firing behaviors above). In the second part we explain what the automation attribute means for firing an agency. The third and fourth part, finally, explain how many objects are read from the preset channels and how many are written to the postset channels when an agency is fired. This once again depends on the firing behavior of the associated activity (compare above).

For the explanation of the firing rule let us assume that $t$ is an agency of a FUNSOFT net $N$ to which the activity $j$ is attached, let $s_{1}, \ldots s_{k}$ be the preset channels of $t$ (all being read via $I N$-edges), let $s_{k+1}, \ldots s_{m}$ be the postset channels of $t$, and let $M_{0}$ be the marking of $N$.

Agency $t$ can be fired if:

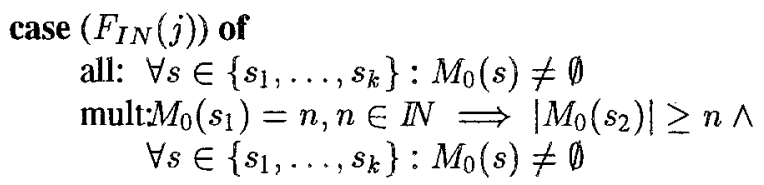

end case

Provided that $t$ can be fired, it actually is fired if:

case (automation mode of $j$ ) of

AUTO: immediately

MAN: as soon as a software developer with the corresponding permission wants to participate in executing $j$

MANY:as soon as all software developers concerned are ready to participate in executing $j$

end case

If $t$ is fired, the successor marking $M_{1}$ of the pre- and postset of $t$ is determined according to the following algorithm. For all other channels of $N$ marking $M_{1}$ equals marking $M_{0}{ }^{5}$.

case $\left(F_{I N}(j)\right)$ of

$$
\begin{gathered}
\text { all: } \forall s \in\left\{s_{1}, \ldots, s_{k}\right\}: \\
\left|M_{1}(s)\right|=\left|M_{0}(s)\right|-1 \\
\operatorname{mult}\left\{M_{0}\left(s_{1}\right)=n, n \in \mathbb{I}\right. \\
\left.\Longrightarrow\left|M_{1}\left(s_{2}\right)\right|=\left|M_{0}\left(s_{2}\right)\right|-n\right) \wedge
\end{gathered}
$$

\footnotetext{
${ }^{5}$ We cannot relate to the values of objects in the following algorithm, since they depend on the activities to be executed. This is the reason for using quantity-restricted coverability trees later on (see section 3.2).
} 


$$
\begin{array}{r}
\forall s \in\left\{s_{1}, \ldots, s_{k}\right\} \backslash\left\{s_{2}\right\}: \\
\left|M_{1}(s)\right|=\left|M_{0}(s)\right|-1
\end{array}
$$

case $\left(F_{\text {OUT }}(j)\right)$ of

$$
\begin{array}{cc}
\text { all: } \quad & \forall s \in\left\{s_{k+1}, \ldots, s_{m}\right\}: \\
& :\left|M_{1}(s)\right|=\left|M_{0}(s)\right|+1 \\
\text { det: } \quad \exists s_{i} \in\left\{s_{k+1}, \ldots, s_{m}\right\}: \\
& \left|M_{1}\left(s_{i}\right)\right|=\left|M_{0}\left(s_{i}\right)\right|+1 \wedge \\
& \forall s \in\left\{s_{k+1}, \ldots, s_{m}\right\} \backslash\left\{s_{i}\right\}: \\
& M_{1}(s)=M_{0}(s) \\
\text { mult: } \quad\left|M_{1}\left(s_{k+1}\right)\right|=\left|M_{0}\left(s_{k+1}\right)\right|+1 \wedge \\
& \left|M_{1}\left(s_{k+2}\right)\right|=\left|M_{0}\left(s_{k+2}\right)\right|+n, \\
& \in \mathbb{N}, \text { where } n \\
& \text { is the integer value } \\
\text { written to } s_{k+1} \\
\forall s \in\left\{s_{k+3}, \ldots, s_{m}\right\}: \\
\left|M_{1}(s)\right|=\left|M_{0}(s)\right|+1
\end{array}
$$

complex: the possible successor markings

are determined by the complex term related to $j$. For a formal definition we refer to [Gru91b].

\section{end case}

Further components of FUNSOFT nets not being discussed in detail in this paper are a set of feedback modifications $M O D$ [DG90] and a set of role identifications and permissions $R$ [Sch91]. A complete definition of FUNSOFT nets can be found in [Gru91b].

\subsection{A FUNSOFT Net Example}

In this section we introduce a FUNSOFT net example. This example is used to illustrate the proposed verification facilities for FUNSOFT nets in the rest of this paper. Parts of this example are taken from the Core Problem [Kat90] and from the software process model describing the development of the Kernel/2r system in the ESF project $\left[\mathrm{ADH}^{+} 92\right]$. The example discussed in this paper consists of 16 (refined and non-refined) agencies and 19 channels.

Figure 3 shows the top level of our example. It sketches the structure of a software development organization. The refined agency Project1 represents the conduction of a single software project. It reads system requirements stored in channel SystemReqs and additional project constraints in channel ProjConstr. As a result, it produces a project 


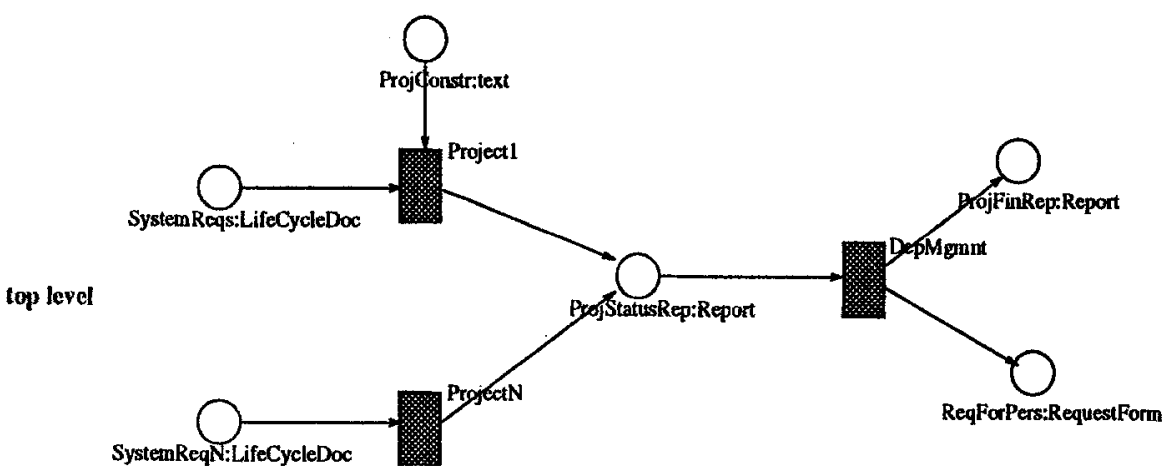

Fig. 3. Top level of the FUNSOFT net example

report (channel ProjStatusRep). Agency ProjectN represents another software project which might be carried out in parallel to the first.

The refined agency DepMgmnt reads the project reports from the various projects (channel ProjStatusRep) and produces finance reports and requests for personnel (channels ProjFinRep and ReqForPers). On this level of abstraction the example shows FUNSOFT nets as a means to integrate multiple projects and higher level management activities.

For simplicity, further description of the example focuses on a single project. Therefore, we restrict ourselves to the refinement of agency Projectl. Figure 4 shows this agency and its refinement. The top part of Figure 4 shows agency Projectl $l$ and its preand postset channels. The bottom part sketches its refinement. The additional, shaded channels SystemReqs, ProjStatusRep and ProjConstr are not part of the refinement. They build the pre- and postset channels of the refined top level agency Projectl. The refinement itself consists of six refined agencies. The refined agencies ReqAna, Design, ModDesAndImpl, Test, and Maintenance represent the lifecycle phases of the software product under development. The agency RiskAssess represents a risk assessment carried out before the project enters the next phase. It reads the project constraints from top level channel ProjConstr and a report from channel Report and writes task descriptions for the various phase agencies to channels ReqTask, DesignTask, ImplTask, TestTask, and MaintTask. Furthermore the agency produces a project status report (channel ProjStatusRep).

We do not present the refinement of each of the refined agencies of this level. For demonstration purposes we present just one of the phase activities in detail. Therefore, we restrict ourselves to the refinement of the agency ModDesAndImpl.

Figure 5 shows agency ModDesAndImpl and its refinement. The agency IdentComps decomposes a document consisting of a list of component specifications stored in channel ImplTask into single component specifications. The results are written to channel CompSpecs. Their number is written to channel NumOfComps.

The agency IdentMods splits a component specification into module specifications stored in channel ModSpecs. Each module is coded by the refined agency EditCompTest. 


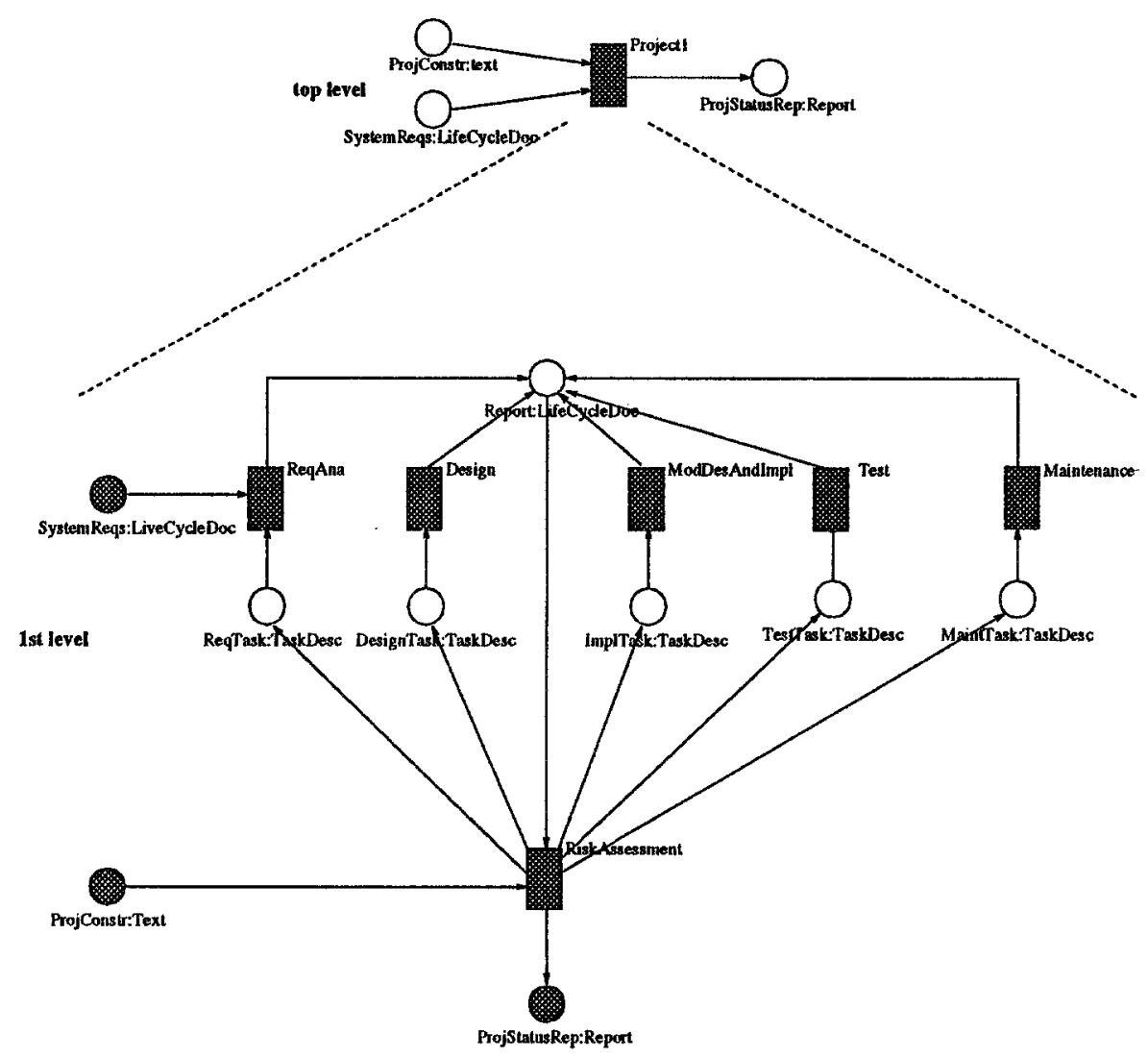

Fig. 4. First refinement level of the FUNSOFT net example

The result is stored in channel ImplMods. The agency BuildListM composes a component out of its building modules. Channel NumOfMods stores the number of modules of the component under development. Channel FreeForWork and the two control flow edges connected synchronize agencies IdentMods and BuildListM. They ensure that all modules being processed by the refined agency EditCompTest at one point of time belong to one component. The agency BuildListC then collects the various components to build the entire system which is stored in channel Report.

Figure 6 shows the refinement of agency EditCompTest. The agency Copy writes a copy of the module specification read from channel ModSpecs to each of its postset channels ModsToImpl and TestSpecs. Agency Code reads a source document from channel ModsToImpl. This either can be a source document with empty source code part (in case of coding of a module from scratch) or a source document with incorrect source code part (in case of rework).

The agency Compile compiles coded modules stored in channel CodedMods and 


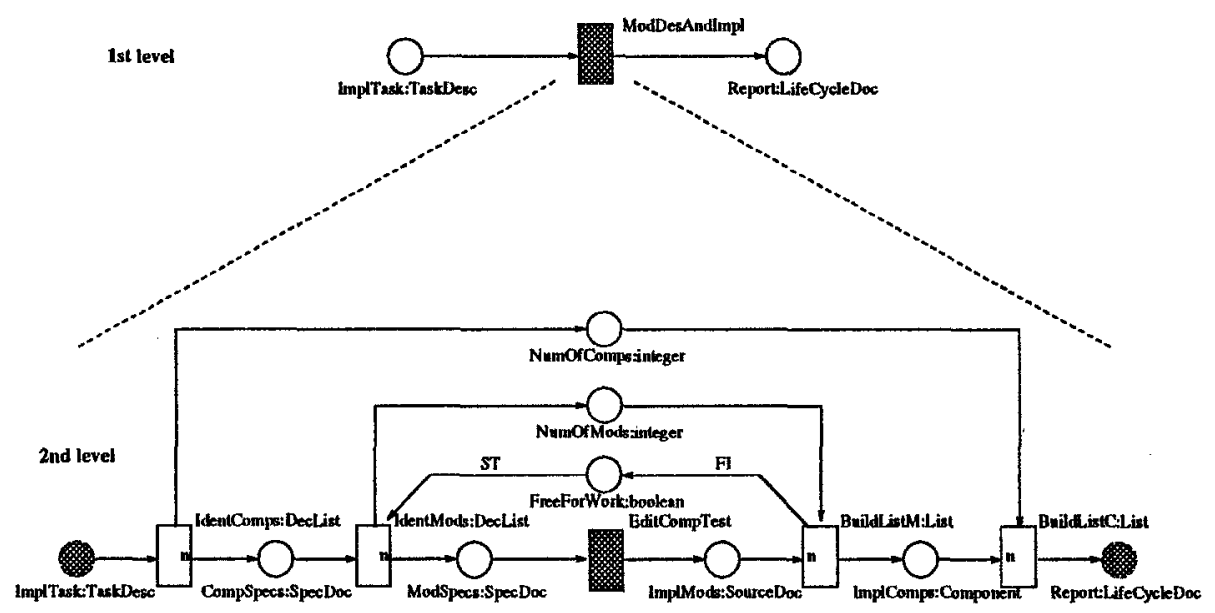

Fig. 5. Second refinement level of the FUNSOFT net example

writes the result to channel CompiledMods. If a compilation is not successful, the coded module is written back to channel ModsTolmpl and a failure report is produced (channel FailureReport).

After the successful compilation of a module, a code review is carried out (represented by agency CodeReview). Accepted modules are written to channel ReviewedMods. In case of rejection, the module is written back to channel ModsToImpl and a rejection form is stored in channel FailureReport.

The agency CreateTPlan reads a module specification and produces a test plan (channel TPlans). The agency Test is used to test single modules according to a predefined test plan. The module to be tested is read from channel ReviewedMods. The test plan is read from channel TPlan. The edge connecting channel TPlan with agency Test has a $\mathrm{CO}$-label. This means that when the test activity is carried out, the test plans remain unchanged in the channel. Agency Test just copies a test plan.

\section{Verification of Software Process Model Properties}

We verify different kinds of software process model properties. We distinguish static and dynamic properties. Static properties of FUNSOFT nets are properties whose investigation is independent of predicting firing sequences of agencies. Dynamic properties are properties that are based on working out potential firing sequences of agencies.

\subsection{Static Software Process Model Properties}

Some of the static properties we investigate are well-known in the world of Petri nets. Others are defined due to their relevance for software process models. An example of a property of the former kind is that of a deadlock. A set of channels $\left\{s_{1}, \ldots, s_{n}\right\}$ is called 

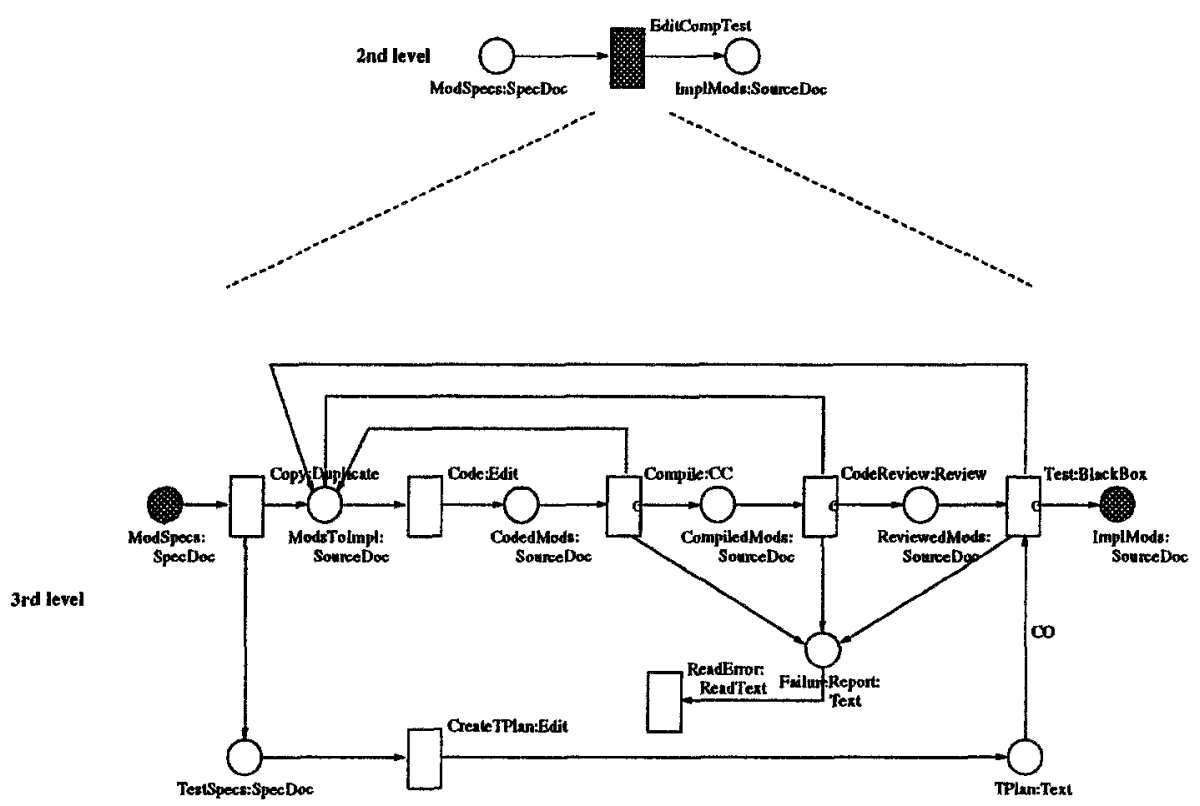

Fig. 6. Third refinement level of the FUNSOFT net example

a deadlock if each agency that writes to a channel $s_{i} \in\left\{s_{1}, \ldots, s_{n}\right\}$, also reads from at least one channel $s_{j} \in\left\{s_{1}, \ldots, s_{n}\right\}$. That means, once the set of channels $\left\{s_{1}, \ldots, s_{n}\right\}$ is unmarked, it remains unmarked.

The notion of FUNSOFT net deadlocks is formally defined as follows.

\section{Definition 3.1 deadlock:}

Let $N$ be a FUNSOFT net. A non-empty set of channels $\left\{s_{1}, \ldots, s_{n}\right\}$ is called a deadlock if the following condition holds

$$
\forall t \in \cup_{i \in\{1, \ldots, n\}} \bullet s_{i}:\left\{s_{1}, \ldots, s_{n}\right\} \cap \bullet t \neq \emptyset
$$

An example of a deadlock are the channels NumOfMods and FreeForWork (Figure 5). Once these channels are unmarked, they remain unmarked, since each agency that writes into one of these channels (agencies IdentMods and BuildListM) also reads from one of these channels. The notion of deadlocks in FUNSOFT nets corresponds to the notion of deadlocks in standard Petri nets. The deadlock \{NumOfMods, FreeForWork\} indicates a modeling error. The control channel FreeForWork is used to enforce that the agency IdentMods can fire again only, if the agency BuildListM has fired before. Since the deadlock is initially unmarked, agency IdentMods never can fire. In order to correct this error, FreeForWork must be initially marked with an object. Then the synchronization mechanism works well from the very beginning.

Another property that is well-known in the world of standard Petri nets is a trap. A trap is a set of channels that remains marked if it is marked once. That means, 
a set of channels $\left\{s_{1}, \ldots, s_{n}\right\}$ is a trap if each agency that reads from a channel $s_{i} \in\left\{s_{1}, \ldots, s_{n}\right\}$ also writes to a channel $s_{j} \in\left\{s_{1}, \ldots, s_{n}\right\}$. Due to the sophisticated output firing behaviors of activities in FUNSOFT nets, the well-known notion of traps had to be adapted for FUNSOFT nets: Let us assume that we want to check if a set of channels $S$ is a trap. Let us, moreover, assume that a $C C$-agency reads and writes to channels of $S$. We recognize that it is not sufficient that one of the postset channels of the $C C$-agency belongs to $S$ in order to be sure that $S$ is a trap (that corresponds to the standard Petri net notion of traps), but that all postset channels of the $C C$-agency have to belong to the trap. Only then it is ensured that $S$ remains marked after firing the $C C$-agency.

The notion of FUNSOFT net traps is formally defined as follows.

\section{Definition 3.2 trap:}

Let $N$ be a FUNSOFT net. A set of channels $\left\{s_{1}, \ldots, s_{n}\right\}$ is called $a$ trap if the following condition holds:

$$
\begin{aligned}
\forall t \in & \cup_{i \in\{1, \ldots, n\}} s_{i} \bullet: \\
& F_{\text {OUT }}\left(T_{J}(t)\right) \in\{\text { det }, \text { some }, \text { complex }\} \Rightarrow t \bullet \subseteq\left\{s_{1}, \ldots, s_{n}\right\} \wedge \\
& F_{\text {OUT }}\left(T_{J}(t)\right) \in\{\text { mult }, \text { all }\} \Rightarrow\left\{s_{1}, \ldots, s_{n}\right\} \cap t \bullet \neq \emptyset
\end{aligned}
$$

If an agency $t$ marks all channels in its postset in each firing (that is the case if $F_{\text {OUT }}\left(T_{J}(t)\right) \in\{$ mult, all $\left.\}\right)$, then it is sufficient that one channel of its postset belongs to the trap, otherwise all channels of its postset must belong to the trap.

Other software process model relevant properties of FUNSOFT nets do not correspond to standard Petri net properties. For example, the notion of useless and unprocessable object types turned out to be useful in software process model verification. These properties do not correspond to standard Petri net properties. An object type is called useless if no object of that type occurs in the initial marking and if no object of that type can be produced.

\section{Definition 3.3 source channel:}

A channel $s$ of a FUNSOFT net $N$ is called a source channel if the following condition holds:

$$
\bullet s=\emptyset
$$

Convention 1 SoC $(N)$ denotes the set of source channels of a FUNSOFT net $N$.

\section{Definition 3.4 useless object type}

Let $N$ be a FUNSOFT net. An object type $o \in O$ is called useless if the following condition holds:

$$
\left\{s \in S \mid S_{T}(s)=o\right\} \subseteq S o C(N) \wedge \forall s \in S: S_{T}(s)=o \Rightarrow M_{0}(s)=\emptyset
$$

Assuming that channels SystemReqs and Report of Figure 5 are initially unmarked, object type LifeCycleDoc is such a useless object type. Channels SystemReqs and Report are the only channels to which object type LifeCycleDoc is attached. Obviously it is an error that LifeCycleDoc is useless, since life cycle documents are vital parts of a 
software process. The error can be removed by adding an initial marking to channel SystemReqs (formally this would be to define $\mid M_{0}$ (SystemReqs) $\mid=1$ ).

An object type is called unprocessable if objects of that type may be produced, but if they are not read by agencies. Such an object type does not necessarily indicate a modeling fault, since final results are not processed intentionally, but a careful look at unprocessable object types helps to detect unprocessable object types which do not represent types of final process results.

\section{Definition 3.5 sink channel:}

A channel $s$ of a FUNSOFT net $N$ is called a sink channel if the following condition holds:

$$
s \bullet=\emptyset
$$

Convention $2 \mathrm{SiC}(N)$ denotes the set of sink channels of a FUNSOFT net $N$.

Definition 3.6 unprocessable object type:

Let $N$ be a FUNSOFT net. An object type $o \in O$ is called unprocessable if it is useless or if the following conditions hold:

$$
\left\{s \in S \mid S_{T}(s)=o\right\} \subseteq \operatorname{SiC}(N)
$$

Further examples of static properties analyzed on the basis of FUNSOFT nets concern activities that produce no results, activities that compete for objects, several activities that produce final results of a software process concurrently, activities that are potentially permanently enabled, and channels that are potentially unbounded. All these properties can be proved by checking the skeletons of FUNSOFT nets (net topology).

\subsection{Dynamic Software Process Model Properties}

Dynamic properties of software process models are properties that depend on predicting which software process states can occur. For example, the channel property of being 10bounded (i.e. the contents of the channel never exceeds 10 objects) depends on predicting software process states. The investigation of that property for channel CompiledMods of the FUNSOFT net shown in Figure 6 clarifies whether a software process state can occur in which more than ten compiled but not reviewed modules exist. Another interesting dynamic property relates to the question of how many activities requiring human interaction at most can be carried out in parallel.

Generally speaking, object values produced in software processes depend on human interaction. If, for example, a software developer participates in firing the Edit-agency, then he determines the contents of the module edited. Neither names nor values of objects produced in software processes are predictable. Accordingly, the investigation of dynamic FUNSOFT net properties is restricted to quantitative questions. A quantitative question does not relate to particular object names and values, but to the number of objects in certain states. The question Can there be ten coded but not compiled modules? is a quantitative one. In contrast to that a question like Can there be ten coded but not compiled modules that use the function display-error? does relate to object values. 
Thus, it cannot be answered a priori. The basis of answering quantitative questions are quantity-restricted coverability trees for FUNSOFT nets.

A node of a quantity-restricted coverability tree of a FUNSOFT net $N$ represents a quantity-restricted marking of $N$. Roughly speaking, a quantity-restricted marking consists of a list of integers, representing the list of numbers of objects per channel. Each edge is labeled with the name of the agency that has to fire in order to transform the marking at the edge origin into the marking at the edge destination. Figure 7 shows two quantity-restricted markings. The top marking identifies a marking under which the first channel is marked with one object, the second channel is unmarked, and so on. By firing agency $t_{1}$, the top marking is transformed into the bottom marking. That means, $t_{1}$ reads an object from the first channel and writes one object to the second and one object to the third channel.

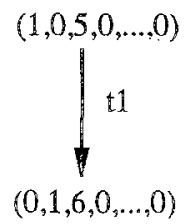

Fig. 7. Constituent parts of a quantity-restricted coverability tree

The way to build quantity-restricted coverability trees is similar to the traditional one [Mur89, HJJJ86]. That means, we keep the representation of the tree finite by covering markings and by cutting subtrees of duplicate markings which occur on the path from the root of the coverability tree to the current marking.

Subsequently we discuss a portion of the quantity-restricted coverability tree for our FUNSOFT net example shown in Figures 3-6 in a little more detail. The portion is shown in Figure 8.

The differences between coverability trees for standard Petri nets and quantityrestricted coverability trees for FUNSOFT nets are discussed below:

\section{storage of synchronization values}

As already mentioned above, an agency with a mult output firing behavior and an agency with a mult input firing behavior are frequently used for synchronization purposes (compare agencies IdentMods and BuildListM of Figure 5). This mechanism ensures that the agency with the mult input firing behavior can only be fired if the required number of objects is available in its first preset channel. When we decide whether such an agency is enabled during building up a coverability tree, we have to know the synchronization value stored in the second preset channel (in Figure 5 this value is stored in channel NumOfMods). For this reason, we cannot abstract from all object values when we build coverability trees. The synchronization values have to be stored. In order to consider all possible cases we specify a range for each activity with a mult output firing behavior. That range indicates the minimal and the 


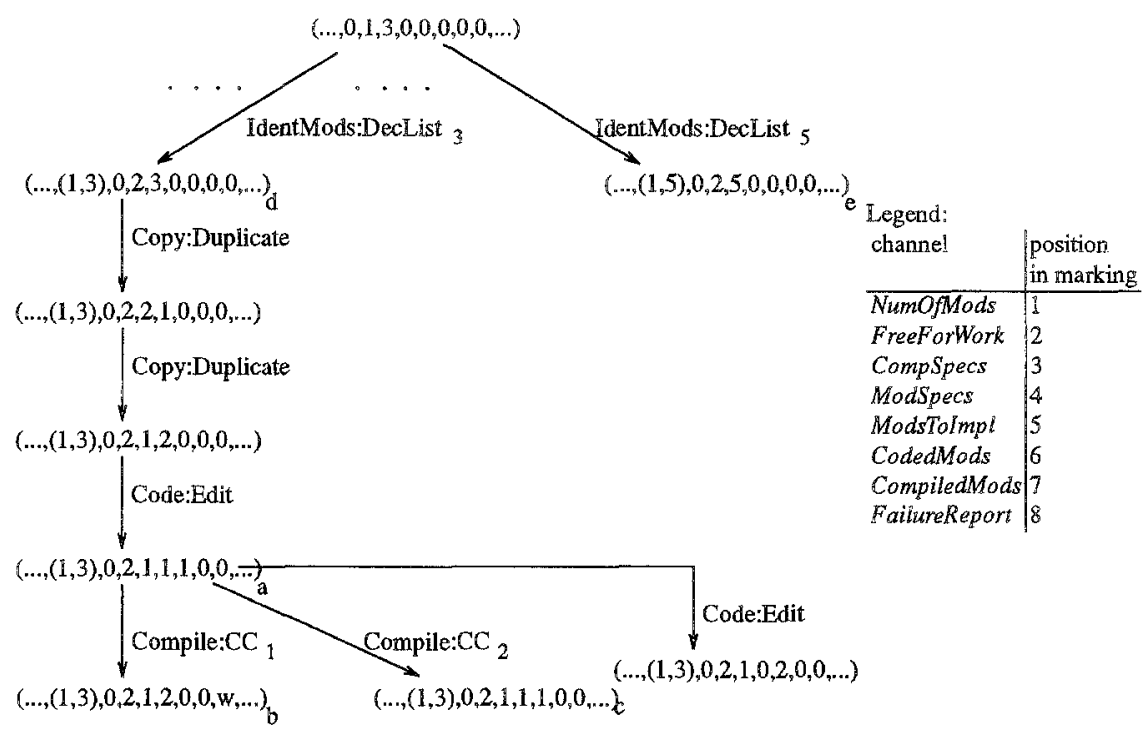

Fig. 8. A quantity-restricted coverability tree

maximal number of objects that can be produced in one execution of the activity. The activity DecList (agency IdentMods of Figure 5), for example, is associated with the range 1 TO 10 . That means, in each firing of agency IdentMods 1 to 10 modules can be written to channel ModSpecs. Accordingly, we consider 10 possible successor markings of a marking under which agency IdentMods is enabled. In the coverability tree of Figure 8 only two of these successor markings are considered (labeled with $d$ and with $e$ ), the others are omitted. The marking labeled with $d$ results from firing agency IdentMods under the assumption that three modules are produced, the marking labeled with $e$ results from firing agency IdentMods under the assumption that five modules are produced. In the marking labeled with $d$, for example, we recognize that the entry for channel NumOfMods is $(1,3)$. That means, channel NumOfMods stores one synchronization integer that has the value 3 .

\section{different results of firing an agency}

Two of the successor of the marking labeled with $a$ in Figure 8 result from firing agency Compile $\left(T_{J}(\right.$ Compile $\left.)=C C\right)$. This, reflects that a $C C$-agency can have two different results. Firstly, a source document which has to be re-edited and a failure report may be produced (represented by the marking labeled with $b$ ) and secondly a successfully compiled source document may be produced (represented by the marking labeled with $c$ ). Since we use activities whose output firing behavior is more sophisticated than the standard Petri net output firing behavior, we have to consider more than one successor marking produced by firing one agency in some cases.

\section{sophisticated notion of covering}

Since we do not abstract from synchronization values, the notion of covered markings is more sophisticated than it is in standard Petri nets. The difference is that a 
FUNSOFT net marking $B$ is said to cover a FUNSOFT net marking $A$ only if each channel stores at least as many objects under marking $B$ as under marking $A$, and if, additionally, each list of synchronization values stored in a synchronization channel under marking B begins with the list of values stored in the same channel under marking A. The corresponding, sophisticated notion of covered markings is defined formally in [Bro91]. The marking labeled with $d$ is covered by the marking labeled with $b$. This is due to the fact that the marking labeled with $b$ contains in each channel at least as many tokens as the marking labeled with $d$. Moreover, channel FailureReport contains one token, while it is unmarked under the marking labeled with $d$. Therefore, arbitrary many tokens can be successively written to channel FailureReport. Thus, an $\omega$ (representing infinite many objects) is found as entry this channel under the marking labeled with $b$.

By inspecting the coverability tree shown in Figure 8 we can, for example, recognize that channel FailureReport is unbounded. That means, arbitrarily many failure reports can be produced. That is because that failure reports do not have to be read before a source document can be re-edited. If a software process modeler wants to enforce that, he can simply introduce a channel to which agency ReadError writes and from which agency Code reads.

Further dynamic software process model properties proved on the basis of quantityrestricted coverability trees concern agencies that are live (an agency $t$ is called live if from each software process state another state can be reached under which that $t$ can be fired) and channels that are unbounded.

The portion of the complete tree shown in Figure 8 shows that the creation and inspection of complete coverability trees cannot be done without tool support due to complexity reasons. This tool and its integration into the software process management environment MELMAC is discussed in the following section.

\section{The Verification Component of MELMAC}

The verification facilities sketched above are implemented in the FUNSOFT net analysis component, called ANAMEL. That component covers validation of software process models, verification of software process model properties, and post-evaluation of software processes. This component is integrated into the software process management environment MELMAC [Gru91a]. MELMAC provides tool support for software process modeling, software process model analysis, and software process model enaction. The MELMAC environment has been used in modeling various examples, for example, in modeling the Core Problem. The experience gathered in modeling and simulating a software process following the Core Problem is described in [Jeg91]. An experience report describing the experience of managing (software) processes with MELMAC can be found in [GJ92].

The ANAMEL component is integrated into MELMAC in a way that enables the use of analysis facilities during the modeling phase. Thus, software process modeling can be arbitrarily interleaved with analyzing the already modeled software process model parts. Thus, the top-down development of hierarchically structured software process models 


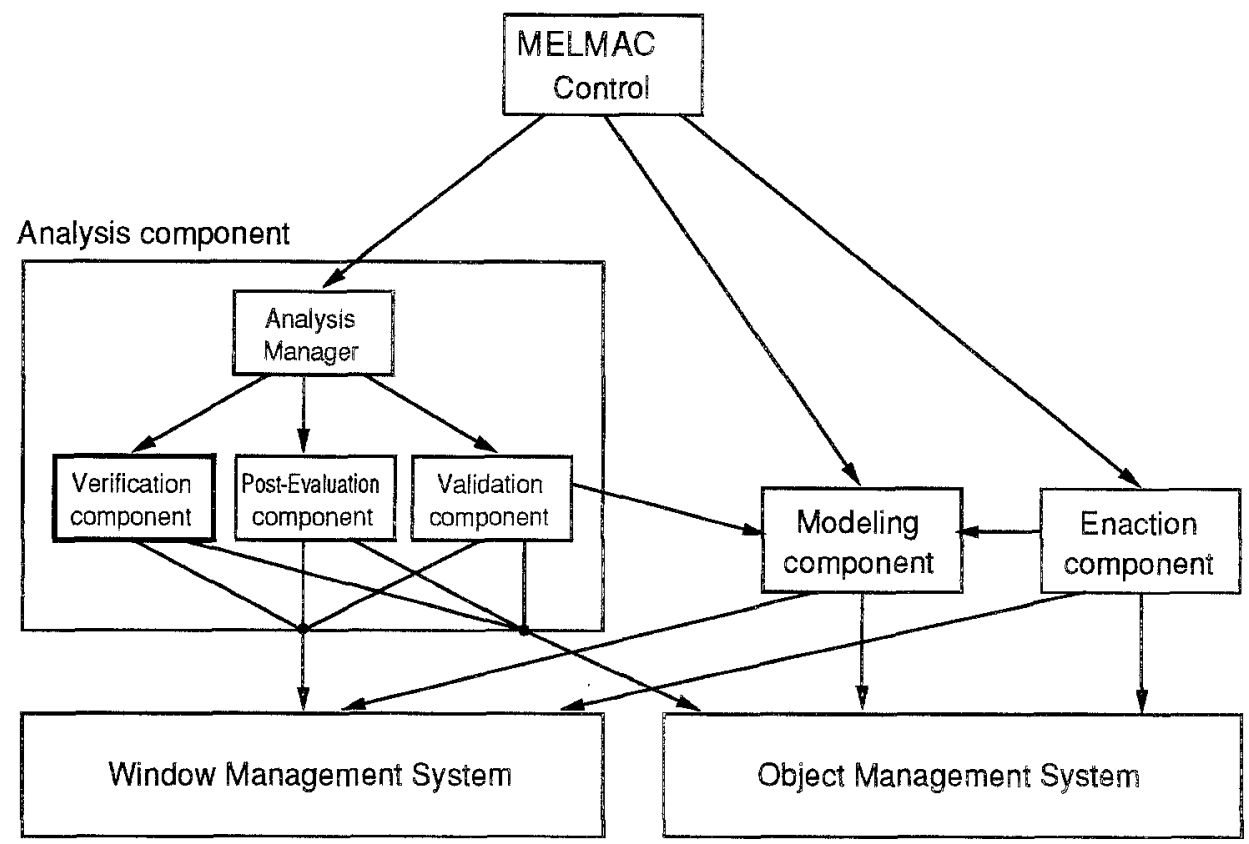

Fig. 9. Architecture of MELMAC

supported by the mechanism of agency refinement is accompanied by analysis facilities that allow analysis on arbitrary levels of abstractions [Gru92]. We thereby enable an incremental analysis of software process models which is well-suited for detecting faults and shortcomings as early as possible.

Corresponding to the focus of this paper we discuss the architecture of the verification component and its embedding into the MELMAC environment.

Figure 9 shows the overall architecture of MELMAC. An arrow between two modules indicates that the source module uses operations provided by the destination module. Thus, arrows represent use-relationships between modules. Figure 9 shows that MELMAC consists of three kinds of component (modeling, analysis, enaction). Due to the focus of this paper the internal structure of the analysis component is sketched. This internal structure reflects the three branches of software process model analysis (verification, post-evaluation, validation). In the following we discuss the components presented in Figure 9 in more detail:

- The module MELMAC Control coordinates the interaction between MELMAC components and controls their start and termination. For example, it ensures that a process model can be enacted only if it has been consistency checked and that a coverability tree can be built only if an initial marking has been defined.

- The Modeling component provides editing facilities for all components of FUNSOFT nets. It contains a syntax-driven and graphical FUNSOFT net skeleton editor, a syntax-driven editor for object types, an editor for the definition of user profiles, 
and some other editors. The Modeling component is called by the Validation component in order to visualize process simulation and by the Enaction component in order to display process states.

- The Enaction component is used to enact a process, to assist and guide software developers, and to record software processes and their simulations.

- The Analysis component implements the analysis facilities for verification of software process model properties, validation of software process models, and postevaluation of software processes. The components implementing these three branches are coordinated by the Analysis manager.

- The Window Management System is used by all MELMAC components which have a user interface. It ensures that all MELMAC components appear in a similar way. Thus, users of MELMAC do not have to get acquainted to different interface styles. Currently, a SunView ${ }^{6}$ and an OpenWindows ${ }^{6}$ implementation of the Window Management System are available.

- The Object Management System stores all information about FUNSOFT nets (object types, net skeletons, activities, markings). We have implemented a set of functions for inserting, updating, and deleting FUNSOFT components as an interface of the Object Management System. Currently, a GRAS [LS88] and an Oracle ${ }^{7}$ implementation of our Object Management System are available.

Figure 10 describes the Verification component of Figure 9 in detail. In the following the components presented in Figure 10 are discussed:

- The module Coverability Tree encapsulates quantity-restricted coverability trees. For the internal representation of these trees, the module Coverability Tree uses the Object Management System. The module Coverability Tree exports operations for retrieval and storage of quantity-restricted markings and their relation (such as store-marking, build-father-marking, retrieve-father-of).

- The module Agency and Marking defines the connection between agencies and quantity-restricted markings. It accesses the FUNSOFT net (stored in the Object Management System) and works out successor markings of a given marking. This depends on FUNSOFT net skeletons and activity firing behaviors. These successor markings are stored by using operations of module Coverability Tree.

- The module Coverability Tree Creation implements the calculation of quantityrestricted coverability trees by using the operations exported by the modules $\mathrm{Cov}$ erability Tree and Agency and Marking. Briefly, module Coverability Tree Creation iterates the operations of Agency and Marking over all nodes and markings of the FUNSOFT net. Thereby, it implements an adapted version of a standard algorithm for the calculation of coverability trees [Mur89, HJJJ86].

- The module Dynamic Property Verification implements the verification of dynamic properties worked out on the basis of quantity-restricted coverability trees. For that purpose it implements, for example, operations that check if a certain agency is live in a certain quantity-restricted coverability tree and what the maximal personnel need for a given quantity-restricted coverability tree looks like.

\footnotetext{
${ }^{6}$ SunView and OpenWindows are trademarks of Sun Microsystems, Incorporated.

${ }^{7}$ Oracle is a trademark of Oracle Corporation.
} 


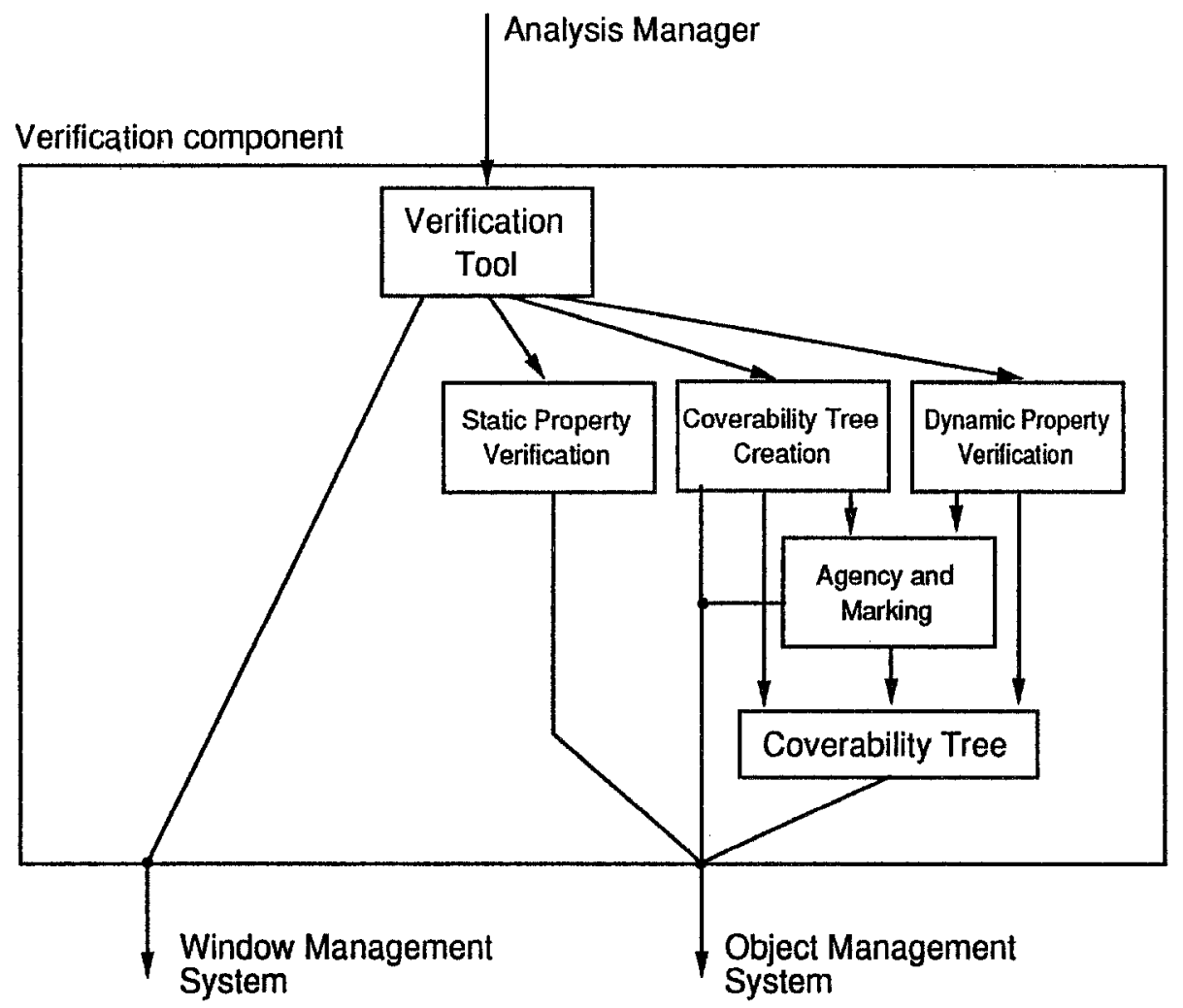

Fig. 10. Architecture of the verification component of MELMAC

- The module Static Property Verification implements the verification of static properties. Since the only basis of verifying static properties is the representation of FUNSOFT nets, module Static Properties uses only operations provided by the Object Management System (where information about FUNSOFT nets is stored).

- The module Verification Control controls the calculation of static and dynamic properties. It manages requests to use already existing quantity-restricted coverability trees or to build up new ones. Moreover, it defines the user interface of the verification component. Therefore, the module Verification Control uses operations provided by the Window Management System module.

Figure 11 shows the user interface of the MELMAC environment after choosing the Verification of static properties branch of the verification component of ANAMEL. In the background we see that we are still able to continue modeling of model parts that are not subject of analysis. In the foreground we see a menu offering different properties that can be verified.

Dynamic properties can be chosen by branching into Verification of dynamic prop- 


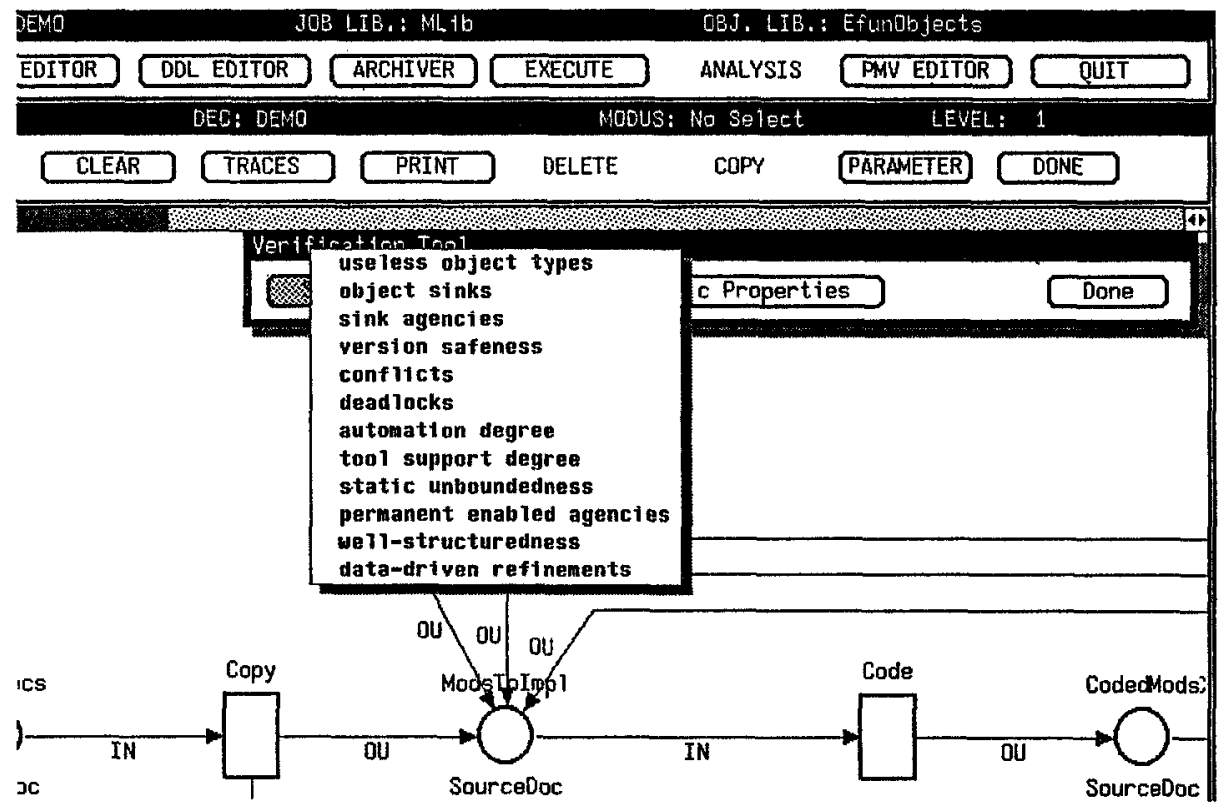

Fig. 11. User Interface of Verification of static properties branch

erties. Figure 12 shows the user interface after choosing that branch.

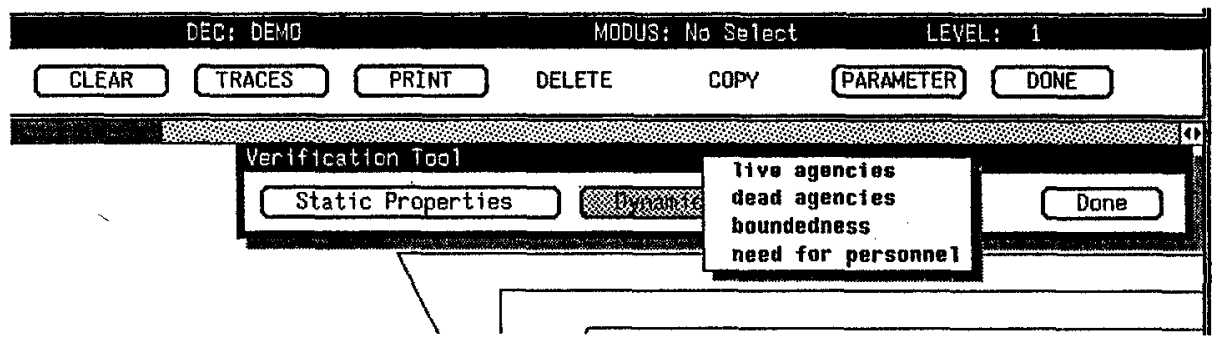

Fig. 12. User Interface of Verification of dynamic properties branch

\section{Conclusion}

In this paper we motivated the need for software process model analysis in general and verification of software process model properties in particular as an integral part of software process management. We discussed the verification facilities for FUNSOFT as they are implemented in the software process management environment MELMAC. 
Our experience with modeling software processes and some other interpersonal processes is that analysis of process models is a valuable help in improving process model quality. Moreover, it turned out that analysis of process models requires tool support. Therefore, our future research efforts will focus on improving the tool support for software process analysis in MELMAC.

Our future research aims at a tool for displaying parts of coverability trees and on a browsing tool for coverability trees. By using such a browsing tool, it would be possible to find all markings with a certain number of objects in a certain channel. Another research topic is the development of a powerful query language for coverability irees. By means of such a query language it would be possible to answer queries like Find all markings which have a successor marking that contains more than five objects in channel $s_{k}$. By extending the analysis facilities in that way we are optimistic that software process model analysis for FUNSOFT nets can be a major help even in analyzing industrial software processes.

In addition to the analysis of software processes, we focus our future application of MELMAC and its analysis facilities also on non-software processes, such as business and administration processes. Our feeling is that the management of these processes promises at least the same benefits as software process management. This feeling is based on the impression that business and administration processes are more strict than software processes. This strictness should make them more amenable to computersupported management and analysis.

Acknowledgements: We want to thank all our colleagues at the chair of Software Technology of Prof. $H$. Weber for various discussions. Moreover, we want to thank the members of the MELMAC group for developing MELMAC. Finally, we want to thank the members of the software engineering department at LION for their cooperation and Chris Lott for his comments on previous versions of this paper.

\section{References}

[ADH ${ }^{+}$92] R. Adomeit, W. Deiters, B. Holtkamp, F. Schülke, and H. Weber. $K / 2_{R}$ A Kernel for the ESF Software Factory Support Environment. In Proceedings of the $2^{\text {nd }}$ International Conference on System Integration, Morristown, New Jersey, June 1992.

[BFG91] S. Bandinelli, A. Fugetta, and C. Ghezzi. Software Processes as Real-Time Systems: A Case Study Using High-Level Petri Nets. In A. Fugetta, R. Conradi, and V. Ambriola, editors, Proceedings of the European Software Process Modeling Workshop, Milan, Italy, May 1991. AICA Press.

[Boe88] B.W. Boehm. A Spiral Model of Software Development and Enhancement. IEEE Computer, 21(5):61-72, May 1988.

[BR87] V.R. Basili and H.D. Rombach. Tailoring The Software Process To Project Goals and Environments. In Proceedings of the $9^{\text {th }}$ International Conference on Software Engineering, Monterey, California, April 1987.

[Bro91] A. Broeckers. Ein Verifikationswerkzeug für FUNSOFT Netze (in German). 1991. Diplomarbeit, University of Dortmund.

[Der92] J.-C. Derniame, editor. Proceedings of the $2^{\text {nd }}$ European Workshop on Software Process Modelling, Trondheim, Norway, September 1992. Springer. Appeared as Lecture Notes in Computer Science 635. 
[DG90] W. Deiters and V. Gruhn. Managing Software Processes in MELMAC. In Proceedings of the Fourth ACM SIGSOFT Symposium on Software Development Environments, pages 193-205, Irvine, California, USA, December 1990. Appeared as ACM Software Engineering Notes, 15(6), December 1990.

[DG91] W. Deiters and V. Gruhn. Software Process Model Analysis Based on FUNSOFT Nets. Mathematical Modeling and Simulation, (8), May 1991.

[Dow86] M. Dowson, editor. Iteration in the Software Process - Proceedings of the $3^{\text {rd }}$ International Software Process Workshop, Beckenridge, Colorado, USA, November 1986.

[Dow91] M. Dowson, editor. "Manufacturing Complex Systems" Proceedings of the $1^{\text {st }}$ International Software Process Conference, Redondo Beach, California, USA, October 1991.

[EG91] W. Emmerich and V. Gruhn. FUNSOFT Nets: A Petri-Net based Software Process Modeling Language. In Proc, of the $6^{\text {th }}$ International Workshop on Software Specification and Design, Como, Italy, September 1991.

[Fug91] A. Fugetta, editor. Proceedings of the $1^{\text {st }}$ European Workshop on Software Process Modelling, Milan, Italy, May 1991. AICA Press.

[Gen87] H.J. Genrich. Predicate/TransitionNets. In W. Brauer, W. Reisig, and G. Rozenberg, editors, Petri Nets: Applications and Relationships to other Models of Concurrency, Berlin, FRG, 1987. Springer. Appeared in Lecture Notes on Computer Science 254.

[GJ92] V. Gruhn and R. Jegelka. An Evaluation of FUNSOFT Nets. In J.-C. Demiame, editor, Software Process Technology - Proceedings of the $2^{\text {nd }}$ European Software Process Modeling Workshop, pages 194-214, Trondheim, Norway, September 1992. Springer. Appeared as Lecture Notes in Computer Science 635.

[Gru] V. Gruhn. Software Process Simulation in MELMAC. Systems Analysis - Modelling - Simulation.

[Gru91a] V. Gruhn. The Software ProcessManagement EnvironmentMELMAC. In A. Fugetta, R. Conradi, and V. Ambriola, editors, Proceedings of the European Software Process Modeling Workshop, Milan, Italy, May 1991. AICA Press.

[Gru91b] V. Gruhn. Validation and Verification of Software Process Models. PhD thesis, University Dortmund, June 1991. Appeared as Technical Report No. 394/91.

[Gru92] V. Gruhn. Software Process Simulation on Arbitrary Levels of Abstraction. In A. Sydow, editor, Computational Systems Analysis 1992, pages 439-444, Amsterdam, The Netherlands, 1992. Elsevier.

[GS92] V. Gruhn and A. Saalmann. Software Process Validation Based on FUNSOFT Nets. In J.-C. Derniame, editor, Software Process Technology - Proceedings of the $2^{\text {nd }}$ European Software Process Modeling Workshop, pages 223-226, Trondheim, Norway, September 1992. Springer. Appeared as Lecture Notes in Computer Science 635.

[Hei91] D. Heimbigner. The Process Modeling Example Problem and its Solutions. In Proceedings of the First International Conference on the Software Process, Redondo Beach, California, USA, October 1991.

[HJJJ86] P. Huber, A.M. Jensen, L.O. Jepsen, and K. Jensen. Reachability Treesfor High-Level Petri Nets. Theoretical Computer Science, (45), 1986.

[HJS89] P. Huber, K. Jensen, and R.M. Shapiro. Hierarchies in Coloured Petri Nets. In Proc. of the $10^{\text {th }}$ Int. Conf. on Application and Theory of Petri Nets, Bonn, FRG, 1989.

[Hum89] W.S. Humphrey. Managing the Software Process. SEI Series in Software Engineering. Addison-Wesley, 1989.

[Jeg91] R. Jegelka. Evaluierung der Software-Prozeßmodellierungs-Sprache FUNSOFTNetze und der Software-Prozeßmanagement-Umgebung MELMAC (in German). 1991. Diplomarbeit, University of Dortmund. 
[Jen83] K. Jensen. High-level Petri nets. Informatik Fachberichte, (66), 1983.

[Jen87] K. Jensen. Coloured Petri Nets. In W. Brauer, W. Reisig, and G. Rozenberg, editors, Petri Nets: Applications and Relationships to other Models of Concurrency, Berlin, FRG, 1987. Springer. Appeared as Lecture Notes on Computer Science 254.

[Kat90] T. Katayama, editor. Proceedings of the $6^{\text {th }}$ International Software Process Workshop, Hakodate, Japan, October 1990.

[Kel88] M.I. Kellner. Representation Formalisms for Software Process Modelling. In Proceedings of the $4^{\text {th }}$ International Software Process Workshop, Moretonhampstead, Devon, UK, May 1988.

[Kel89] M.I. Kellner. Software Process Modeling Example. In Proceedings of the $5^{\text {th }}$ International Software Process Workshop, Kennebunkport, Maine, USA, September 1989.

[Leh87] M. Lehman. Process Models, Process Programs, Programming Support - Invited Response To A Keynote Address By Lee Osterweil. In Proceedings of the $9^{\text {th }}$ International Conference on Software Engineering, Monterey, California, 1987.

[LH89] L. Liu and E. Horowitz. A Formal Model for Software Project Management. IEEE Transactions on Software Engineering, 15(10), October 1989.

[LS88] C. Lewerentz and A. Schuerr. GRAS - a Management System for Graph - like Documents. In Proc. 3rd Int. Conf. on Data and Knowledge Bases, Jerusalem, 1988. Morgan Kaufmann Publishers Inc.

[Luq89] Luqi. Software Evolution ThroughRapid Prototyping. IEEE Compuier, 22(5):13-25, May 1989.

[Mur89] T. Murata. Petri Nets: Properties, Analysis and Applications. Proceedings of the IEEE, 77(4), April 1989.

[Ost87] L. Osterweil. Software Processes are Software Too. In Proceedings of the $9^{\text {th }}$ International Conference on Software Engineering, Monterey, California, April 1987.

[Per91] D.E. Perry, editor. Proceedings of the $7^{\text {th }}$ International Software Process Workshop, Yountville, California, USA, October 1991.

[Rei86] W. Reisig. Petrinetze (in German). Springer, Berlin, FRG, 1986.

[Roy70] W.W. Royce. Managing the development of large software systems: Concepts and techniques. In Proc. WESCON, 1970.

[Sch91] U. Schindler. Projekt Management in Software Prozeßmodellen (in German). 1991. Diplomarbeit, University of Dortmund.

[Som92] I. Sommerville. Software Engineering. Addison-Wesley, fourth edition, 1992. 